\title{
Individual Differences in Nucleus Accumbens Activity to Food and Sexual Images Predict Weight Gain and Sexual Behavior
}

\author{
Kathryn E. Demos, ${ }^{1,2}$ Todd F. Heatherton, ${ }^{1}$ and William M. Kelley ${ }^{1}$ \\ ${ }^{1}$ Department of Psychological and Brain Sciences, Center for Cognitive Neuroscience, Dartmouth College, Hanover, New Hampshire 03755, and \\ ${ }^{2}$ Department of Psychiatry and Human Behavior, Weight Control and Diabetes Research Center, The Miriam Hospital and Alpert Medical School of Brown \\ University, Providence, Rhode Island 02903
}

Failures of self-regulation are common, leading to many of the most vexing problems facing contemporary society, from overeating and obesity to impulsive sexual behavior and STDs. One reason that people may be prone to engaging in unwanted behaviors is heightened sensitivity to cues related to those behaviors; people may overeat because of hyperresponsiveness to food cues, addicts may relapse following exposure to their drug of choice, and some people might engage in impulsive sexual activity because they are easily aroused by erotic stimuli. An open question is the extent to which individual differences in neural cue reactivity relate to actual behavioral outcomes. Here we show that individual differences in human reward-related brain activity in the nucleus accumbens to food and sexual images predict subsequent weight gain and sexual activity 6 months later. These findings suggest that heightened reward responsivity in the brain to food and sexual cues is associated with indulgence in overeating and sexual activity, respectively, and provide evidence for a common neural mechanism associated with appetitive behaviors.

\section{Introduction}

Although humans have an impressive capacity for self-regulation, failures are common, leading to many preventable health problems, from overeating and obesity to impulsive sexual behavior and HIV/ AIDS. One reason people may engage in such behaviors is heightened sensitivity to environmental cues (Heatherton, 2011). Neuroimaging studies assessing reactivity to appetitive cues have consistently observed activation of human reward circuitry, including ventral striatum and amygdala (Passamonti et al., 2009; Demos et al., 2011). For example, obese women exhibit greater activity in the nucleus accumbens (NAcc) in response to high-calorie food items than do healthy-weight control subjects (Stoeckel et al., 2008). Women with bulimia nervosa also show increased brain reward activity when observing food cues (Brooks et al., 2011). Likewise, cues of physical attractiveness activate NAcc in both men and women (Cloutier et al., 2008), as does exposure to erotic images (Hamann et al., 2004).

Neural and physiological reactivity to drug cues predicts important behavioral outcomes, such as smoking cessation (Janes et al., 2010) and cocaine relapse (Back et al., 2010). Does such reac-

Received Nov. 14, 2011; revised March 2, 2012; accepted March 5, 2012.

Author contributions: K.E.D., T.F.H., and W.M.K. designed research; K.E.D. performed research; K.E.D. and W.M.K. analyzed data; K.E.D., T.F.H., and W.M.K. wrote the paper.

This work was supported by the National Institute of Drug Abuse (DA022582). We thank Cary Savage, Paul Whalen, Courtney Rogers, Tammy Moran, Leah Somerville, Kristina Caudle, and Dylan Wagner for their assistance on this project.

Correspondence should be addressed to Dr. Kathryn E. Demos, Weight Control and Diabetes Research Center, The Miriam Hospital, Brown University Medical School, 196 Richmond Street, Providence, RI 02903. E-mail: Kathryn_demos@brown.edu.

DOI:10.1523/JNEUROSCI.5958-11.2012

Copyright $\odot 2012$ the authors $\quad 0270-6474 / 12 / 325549-04 \$ 15.00 / 0$ tivity predict long-term outcomes for non-drug behaviors? An open question is whether individual differences in brain activity, specifically in regions associated with reward motivation, are associated with an increased proclivity for indulgence and thereby predict future weight gain and/or sexual promiscuity. We hypothesized that individual differences in NAcc activity in response to viewing food items and sexual scenes would predict future weight gain and sexual behavior.

Shortly after arrival on campus, a cohort of first-year female college students $(N=58)$ were scanned using functional magnetic resonance imaging (fMRI) while viewing images of animals, environmental scenes, appetizing food items, and people (Fig. 1). Some of the images of people depicted people in sexual scenes or consuming alcohol. Task instructions were simply to indicate whether a person was present in the image. Before scanning, participants were weighed under the guise that it was a necessary step in the scanning procedure. As such, participants were naive to the goals of the study. Six months later, 48 of the participants returned to the laboratory where they were again weighed, and then completed questionnaires assessing their sexual behavior. It was hypothesized that activity in the NAcc would reflect individual differences in appetitive behaviors (i.e., eating and sex).

\section{Materials and Methods \\ Subjects}

Fifty-eight matriculating native English-speaking females in their first year of college at Dartmouth College participated in this experiment. Of the 58, 48 returned 6 months later for a follow-up behavioral session; thus, only 10 participants were lost to follow-up (i.e., did not respond to requests to return) at 6 months. Participants were between the ages of 18 and 19 (mean age $=18$ years). No subject reported abnormal neurolog- 
ical history and all had normal or corrected-tonormal visual acuity. Each subject provided informed consent in accordance with the guidelines set by the Committee for the Protection of Human Subjects at Dartmouth College and received monetary compensation for participating in this study.

\section{Apparatus}

Imaging was performed on a Philips Intera Achieva 3-tesla scanner (Phillips Medical Systems) with a SENSE (SENSEitivity Encoding) head coil. During scanning, visual stimuli were generated with an Apple MacBook Pro Laptop computer running SuperLab 4.0 software (Cedrus Corporation). An Epson (model ELP7000) LCD projector was used to display stimuli on a screen positioned at the head-end of the scanner bore and subjects viewed it through a mirror mounted on top of the head coil. A fiber optic, light-sensitive key press interfacing with the Cedrus Lumina Box recorded subjects' responses. Cushions were placed around the head to minimize movement during scanning and increase comfort.

\section{Imaging}

Anatomic images were acquired using a high-

resolution 3-D magnetization-prepared rapid gradient echo sequence (60 sagittal slices; TE, $4.6 \mathrm{~ms}$; TR, $9.9 \mathrm{~ms}$; flip angle, $8^{\circ}$; voxel size, $1 \times 1 \times$ $1 \mathrm{~mm}$ ). Functional images were collected using T2* fast field echo, echo planar functional imaging sensitive to BOLD contrast (TR, $2500 \mathrm{~ms}$; TE, $35 \mathrm{~ms}$; flip angle, $90^{\circ} ; 3 \times 3 \mathrm{~mm}$ in-plane resolution; sense factor of 2 ). Functional scanning was performed in five runs; during each run, 144 axial images (36 slices, $3.5 \mathrm{~mm}$ slice thickness, $0.5 \mathrm{~mm}$ skip between slices) were acquired, allowing complete brain coverage.

\section{Procedure}

Time 1. Participants $(n=58)$ were scheduled for an fMRI session within the first month of their arrival on the college campus. Participants were asked to refrain from eating, consuming alcohol or caffeine, and smoking for $2 \mathrm{~h}$ before their fMRI session. Immediately before scanning, each subject was asked to respond to a set of questions to assess their current state, wherein they listed food and drink consumption, activity level, and current hunger level.

Critically, in addition to participants' self-report of their weight (a standard procedure for fMRI scanning), these participants were weighed and measured using a calibrated Detecto Physician's Scale (Cardinal Scale Manufacturing) immediately before scanning. To explain the need for obtaining these anthropometrics, participants were told of the importance of having an accurate reading of body measurements for fMRI scanning. These measurements were used to calculate Body Mass Index [BMI; (weight in kg)/(height in $\mathrm{m}^{2}$ ] and served as a baseline (Time 1) assessment of BMI to be used in comparison with those obtained at Time 2 (6 months later).

Subjects were then scanned in an event-related cue-reactivity paradigm while viewing images of animals (80), food (80), people drinking alcohol (80), people in sexual scenes (80), people (nonsexual/nondrinking) (80), and environmental scenes (80) (Fig. 1). The images were compiled from the Internet, as well as the International Affective Picture System (Lang et al., 2008) and scaled to 480 by 360 pixels in size using Adobe Photoshop 7.0. During scanning, subjects were asked to simply determine whether or not each image contained a person and to use key presses to make their responses (left-handed response for "nonperson"; right-handed response for "person"). The purpose of this task was to ensure subjects' attention to the images. Each image was presented for $2000 \mathrm{~ms}$, followed by a crosshair $(500 \mathrm{~ms})$, and randomly intermixed across the trial with jittered periods of fixation $(0-10,000 \mathrm{~ms}$; mean intertrial interval $=5000 \mathrm{~ms})$.

Time 2 (6-month follow-up). Approximately 6 months after Time 1, the same 58 participants were contacted and asked to return to the lab for a behavioral follow-up session. A total of 48 participants returned to participate in this session. The mean time between sessions was 180.5 days (range, 160-200 d). During this session, the Sociosexual Orientation Inventory (Simpson and Gangested, 1991) and the Sexual Desire Inventory (SDI) (Spector et al., 1996) were administered. Following completion of the questionnaires, participants were again weighed and measured on the same calibrated medical scale used at Time 1 .

\section{fMRI data analyses}

fMRI data were analyzed using Statistical Parametric Mapping software (SPM2; Wellcome Department of Cognitive Neurology, London, UK) (Friston et al., 1995). For each functional run, data were preprocessed to remove sources of noise and artifact. Functional data were realigned within and across runs to correct for head movement, coregistered with each participant's anatomic data, and transformed into a standard anatomic space ( $3 \mathrm{~mm}$ isotropic voxels) based on the ICBM 152 brain template (Montreal Neurological Institute). Normalized data were then spatially smoothed ( $6 \mathrm{~mm}$ full-width at half-maximum) using a Gaussian kernel, and globally scaled to permit between group comparisons. Analyses of fMRI data included: formation of statistical contrast images, regional analysis of hemodynamic responses using an anatomically defined regions of interest (ROI) for the nucleus accumbens, and exploratory whole-brain regression analysis.

\section{Statistical images}

For each participant, a general linear model incorporating task effects (modeled as an event-related function convolved with the canonical hemodynamic response function), a mean, and a linear trend were used to compute $t$-contrast images (weighted parameter estimates) for each comparison at each voxel. These individual contrast images were then submitted to a second-level, random-effects analysis to create mean $t$-images (thresholded at $p=0.001$, uncorrected).

\section{ROI analyses}

This study sought to investigate individual differences in reward processing during simple viewing of food stimuli and sexual scenes. To specifically explore the involvement of the NAcc, a hub of the dopaminergic reward system consistently identified in human and animal studies of reward, addiction, and motivation, ROIs were created based on anatomical coordinates defined in a stereotactic investigation of the human NAcc (Neto et al., 2008). Spherical ROIs (6 mm) were built centered at MNI coordinates $x=-9, y=6, z=-4$ (left NAcc) as well as $x=9, y=$ $6, z=-4$ (right NAcc). Signal intensities (beta values) for this ROI were 

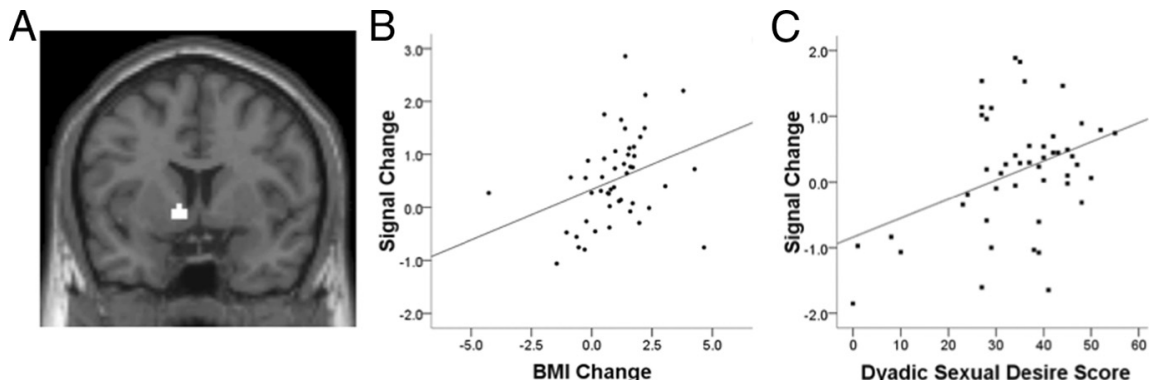

Figure 2. Nucleus accumbens activity to food and sexual images predicts weight gain and sexual desire, respectively. $A$, The anatomical ROI of the left NAcc with central Talairach coordinates $(x, y, z)-9,6,-4$ is displayed on a coronal brain slice. $\boldsymbol{B}$, Signal change in the left NAcc (anatomically defined ROI) in response to viewing food images at Time 1 positively correlated with change in BMI 6 months later (Time $2-$ Time 1) $(r=0.37, p<0.05)$. C, Signal change in the left NAcc ROI in response to viewing sexual scenes at Time 1 positively correlated with dyadic sexual desire $(r=0.39, p<0.01)$. A similar correlation was observed between NAcc activity to sexual scenes and solitary sexual desire (data not shown; $r=0.36, p=0.01$ ).

then calculated separately for each condition compared with fixation baseline and the relationship between activity in this area and behavioral measures were examined statistically.

\section{Exploratory whole-brain regression analyses}

Although the primary analyses reported here were based on an a priori defined region of interest centered on the NAcc, to identify additional activations that showed a relationship between appetitive behavior and brain activity, we performed additional exploratory whole-brain regression analyses. To identify regions associated with weight change, BMI change for each subject was entered into a whole-brain regression analysis examining the neural response to food images (food $>$ baseline). Similarly, to identify regions whose activity in response to sexual scenes was associated with sexual behavior, SDI scores (dyadic and solitary) for each subject were entered into a whole-brain regression analysis examining the neural response to sexual images (sexual scenes $>$ baseline).

\section{Results}

\section{Predicting weight change}

Anatomical ROIs were selected for both the left and right NAcc based on stereotactic coordinates $(x, y, z=+/-9,6,-4$; Fig. $2 A$ ). Activity in response to viewing food images (during scanning; Time 1) was then used to predict weight change $\sim 6$ months later (Time 2; calculated as BMI at Time 2 - BMI at Time 1). Results revealed that activity in the left NAcc correlated positively with BMI change, such that greater activity predicted subsequent weight gain $(r=0.37, p<0.05$; Fig. $2 B)$. Importantly, this relationship was unique to the food images. NAcc activity in response to the non-food images did not predict weight gain (animals: $r=$ -0.07 ; people: $r=-0.17$; drinking scenes: $r=0.01$; sexual scenes: $r=-0.26$; environmental scenes: $r=-0.03$ ). When contrasted directly (using standard formulas for contrasting $r$ values using Fishers $\mathrm{r}$ to $z$ transformation), the food cuereactivity correlation with BMI change differed from the correlation for nonfood images $(p<0.01)$.

The relationship between left NAcc (INAcc) activity and weight change was also evident in whole-brain regression analyses. Results of whole-brain regression analyses revealed that activity in the $\operatorname{lNAcc}(-9,9,-3)$ was the only region to show a significant correlation with BMI at this threshold. Using a more lenient statistical threshold $(p<0.005)$, additional positive correlations were observed in the anterior cingulate (BA 32: 6, 50, 0 ) and the inferior temporal gyrus (BA 20:36, $-5,-33$ ).

\section{Predicting sexual behavior}

Just as cue reactivity to food images was investigated as potential predictors of weight gain, cue reactivity to sexual images was used to predict sexual desire (as indexed by the SDI: solitary sexual desire, which measures interest in behaving sexually by oneself, and dyadic sexual desire, which measures interest in behaving sexually with a partner) (Spector et al., 1996). Left NAcc activity in response to viewing sexual scenes was positively correlated with both solitary sexual desire $(r=0.36, p=$ $0.01)$ and dyadic sexual desire $(r=0.39$, $p<0.01$; Fig. $2 C$ ). Whereas the relationship between weight gain and NAcc activity was unique to food images, the relationship between sexual desire and NAcc activity was unique to sexual images. None of the other image categories predicted sexual desire (all $r$ 's $< \pm 0.20$ ) and the correlation between cue reactivity for sexual scenes and SDI scores differed from nonsexual images correlations $(p<0.05)$.

Results of the whole-brain exploratory analysis revealed additional positive correlations between cue reactivity to sexual scenes and the solitary and dyadic sexual desire measures (thresholded at $p<0.001$, minimum cluster size $=5$ voxels $)$ in a region of the orbitofrontal cortex (BA 10: 15, 58, 0), right middle temporal gyrus (BA 20:39, 2, -20), and left parahippocampal gyrus $(-21,-27,-16)$.

Although the number of sexual partners that participants reported was anticipated to be used as a continuous variable, 26 of the 48 returning participants reported having no sexual partners. Thus, because more than half of the participants reported no sexual activity during the year, sexual behavior was treated as a discrete variable [individuals who had sex during the year $(N=$ $22)$ vs those who did not $(N=26)]$. Activity in the left NAcc while viewing sexual images was greater in individuals who reported sexual activity with at least one partner $\left(t_{(46)}=6.30, p<0.0001\right)$ Left NAcc activity did not differ between groups for any of the other image cues (all $p$ 's $>0.14$ ).

\section{Discussion}

The present study demonstrates that reward activity in response to appetitive cues predicts long-term and consequential behavioral outcomes. According to both human and animal studies, exposure to appetitive cues increases the likelihood that a craved substance will be consumed or a behavior enacted (Stewart et al., 1984; Drummond et al., 1990; Jansen, 1998). Recent work by Kober et al. (2010) revealed that cognitive strategies aimed at reducing cravings for cigarettes and food were accompanied by reduced activity in the ventral striatum. Martin and Delgado (2011) demonstrated similar striatal activity reductions during successfully regulated monetary decisions, suggesting perhaps that active self-regulation may be associated with reduced reward sensitivity (Kober et al., 2010; Martin and Delgado, 2011). Imaging studies suggest that self-regulation involves a balance between subcortical brain regions representing the reward, salience and emotional value of a stimulus, and frontoparietal regions associated with executive control (Heatherton and Wagner, 2011), particularly dorsolateral prefrontal cortex (Hare et al., 2009; Staudinger et al., 2011). Collectively, these findings suggest a domain-general pattern for reward-related brain activity whereby heightened activity in the nucleus accumbens may be used as a proxy for future appetitive behaviors and reduction of such behaviors may involve higher-order cognitive systems. Moreover, there is some evidence that genetic 
variations may differentially affect the relationship between reward activity and weight (Stice et al., 2010).

It seems likely that the stress associated with the first year of college may be a contributing factor to changes in behavior. Research in nonhuman animals indicates that stress weakens prefrontal control over behavior (Arnsten, 2009) while also sensitizing reward systems to biologically salient stimuli (Piazza and Le Moal, 1998). Thus, stress may disrupt self-regulatory efforts because it is associated with reduced top-down control from prefrontal cortex in conjunction with heightened hedonic responses to appetitive stimuli (Heatherton and Wagner, 2011). For instance, a considerable amount of research demonstrates that stress and other types of negative affect produce increases in eating, particularly for those trying to control their weight (Heatherton and Baumeister, 1991). Other factors, such as less frequent exercise, living away from parental influence, and changes in daily routines and sleep and their hormonal consequences may all be contributing factors to weight gain and changes in sexual behavior observed among college students. Future research is needed to disentangle these putative mechanisms.

As noted, cue exposure increases activity in brain reward regions, but it also produces spontaneous activity in the action observation network, such as when smokers spontaneously engage action representation brain areas when viewing others smoking (Wagner et al., 2011). According to contemporary research in social cognition, exposure to appetitive cues primes behavior outside of people's intentions or awareness (Bargh and Morsella, 2008). The putative mechanism for behavioral priming is that cue exposure activates mental representations that lead to unconscious goal pursuit, which ultimately produces the same outcomes that consciously pursuing goals does (Dijksterhuis et al., 2007). Indeed, even if people are aware of being exposed to appetitive cues, they may be unaware of the effect such cues have on their behavior (Stacy and Wiers, 2010). From this perspective, exposure to food or sexual images activates mental representations of their attendant behaviors and activates unconscious goals to engage in those behaviors.

The implication of the current research is that there are individual differences in the extent to which cue exposure primes behavior, such that some individuals show more robust reward activity to appetitive cues, which in turn may produce greater behavioral priming. It is important to note that cue reactivity in the current study shows material specificity, such that those who showed heightened reward activity to food images gained weight but did not engage in more sexual behavior, and vice versa. Understanding how individual differences in reward responsivity primes specific actions that have measurable long-term behavioral outcomes may assist in the development of more effective strategies for preventing self-regulation failures, such as overeating or engaging in risky sexual activity. This research shows that there are important long-term consequences of individual differences in selective cue responsivity to appetitive cues.

\section{References}

Arnsten AF (2009) Stress signaling pathways that impair prefrontal cortex structure and function. Nat Rev Neurosci 10:410-422.

Back SE, Hartwell K, DeSantis SM, Saladin M, McRae-Clark AL, Price KL, Moran-Santa Maria MM, Baker NL, Spratt E, Kreek MJ, Brady KT (2010) Reactivity to laboratory stress provocation predicts relapse to cocaine. Drug Alcohol Depend 106:21-27.

Bargh JA, Morsella E (2008) The unconscious mind. Perspect Psychol Sci 3:73-79.

Brooks SJ, O’Daly OG, Uher R, Friederich HC, Giampietro V, Brammer M, Williams SC, Schiöth HB, Treasure J, Campbell IC (2011) Differential neural responses to food images in women with bulimia versus anorexia nervosa. PLoS One 6:e22259.
Cloutier J, Heatherton TF, Whalen PJ, Kelley WM (2008) Are attractive people rewarding? Sex differences in the neural substrates of facial attractiveness. J Cogn Neurosci 20:941-951.

Demos KE, Kelley WM, Heatherton TF (2011) Dietary restraint violations influence reward responses in nucleus accumbens and amygdala. J Cogn Neurosci 23:1952-1963.

Dijksterhuis A, Chartrand TL, Aarts H (2007) Effects of priming and perception on social behavior and goal pursuit. In: Social psychology and the unconscious: the automaticity of higher mental processes (Bargh JA, ed), pp 51-132. Philadelphia: Psychology.

Drummond DC, Cooper T, Glautier SP (1990) Conditioned learning in alcohol dependence: implications for cue exposure treatment. Br J Addict 85:725-743

Friston KJ, Holmes AP, Worsley KJ, Poline JB, Frith CD, Frackowiak RS (1995) Statistical parametric maps in functional imaging: a general linear approach. Hum Brain Mapp 2:189-210.

Hamann S, Herman RA, Nolan CL, Wallen K (2004) Men and women differ in amygdala response to visual sexual stimuli. Nat Neurosci 7:411-416.

Hare TA, Camerer CF, Rangel A (2009) Self-control in decision-making involves modulation of the vmPFC valuation system. Science 324:646-648.

Heatherton TF (2011) Neuroscience of self and self-regulation. Annu Rev Psychol 62:363-390.

Heatherton TF, Baumeister RF (1991) Binge eating as escape from selfawareness. Psychol Bull 110:86-108.

Heatherton TF, Wagner DD (2011) Cognitive neuroscience of selfregulation failure. Trends Cogn Sci 15:132-139.

Janes AC, Pizzagalli DA, Richardt S, deB Frederick B, Chuzi S, Pachas G, Culhane MA, Holmes AJ, Fava M, Evins AE, Kaufman MJ (2010) Brain reactivity to smoking cues prior to smoking cessation predicts ability to maintain tobacco abstinence. Biol Psychiatry 67:722-729.

Jansen A (1998) A learning model of binge eating: cue reactivity and cue exposure. Behav Res Ther 36:257-272.

Kober H, Mende-Siedlecki P, Kross EF, Weber J, Mischel W, Hart CL, Ochsner KN (2010) Prefrontal-striatal pathway underlies cognitive regulation of craving. Proc Natl Acad Sci U S A 107:14811-14816.

Lang PJ, Bradley MM, Cuthbert BN (2008) International affective picture system (IAPS): affective ratings of pictures and instruction manual. Technical Report A-8. Gainesville, FL: University of Florida.

Martin LN, Delgado MR (2011) The influence of emotion regulation on decision making under risk. J Cogn Neurosci 23:2569-2581.

Neto LL, Oliveira E, Correia F, Ferreira AG (2008) The human nucleus accumbens: where is it? A stereotactic, anatomical and magnetic resonance imaging study. Neuromodulation 11:13-22.

Passamonti L, Rowe JB, Schwarzbauer C, Ewbank MP, von dem Hagen E, Calder AJ (2009) Personality predicts the brain's response to viewing appetizing foods: the neural basis of a risk factor for overeating. J Neurosci 29:43-51.

Piazza PV, Le Moal M (1998) The role of stress in drug self-administration. Trends Pharmacol Sci 19:67-74.

Simpson JA, Gangestad SW (1991) Individual differences in sociosexuality: evidence for convergent and discriminant validity. J Pers Soc Psychol 60:870-883.

Spector IP, Carey MP, Steinberg L (1996) The sexual desire inventory: development, factor structure, and evidence of reliability. J Sex Marital Ther 22:175-190.

Stacy AW, Wiers RW (2010) Implicit cognition and addiction: a tool for explaining paradoxical behavior. Annu Rev Clin Psychol 6:551-575.

Staudinger MR, Erk S, Walter H (2011) Dorsolateral prefrontal cortex modulates striatal reward encoding during reappraisal of reward anticipation. Cereb Cortex 21:2578-2588.

Stewart J, de Wit H, Eikelboom R (1984) Role of unconditioned and conditioned drug effects in the self-administration of opiates and stimulants. Psychol Rev 91:251-268.

Stice E, Yokum S, Bohon C, Marti N, Smolen A (2010) Reward circuitry responsivity to food predicts future increases in body mass: moderating effects of DRD2 and DRD4. Neuroimage 50:1618-1625.

Stoeckel LE, Weller RE, Cook EW 3rd, Twieg DB, Knowlton RC, Cox JE (2008) Widespread reward-system activation in obese women in response to pictures of high-calorie foods. Neuroimage 41:636-647.

Wagner DD, Dal Cin S, Sargent JD, Kelley WM, Heatherton TF (2011) Spontaneous action representation in smokers when watching movie characters smoke. J Neurosci 31:894-898. 